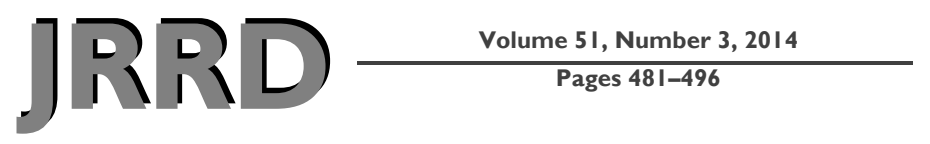

\title{
Posture-dependent control of stimulation in standing neuroprosthesis: Simulation feasibility study
}

\author{
Musa L. Audu, PhD; ' Steven J. Gartman, MS; Raviraj Nataraj, PhD; Ronald J. Triolo, PhD \\ Departments of Biomedical Engineering and Orthopedics, Case Western Reserve University, Cleveland, OH; Louis Stokes \\ Cleveland Department of Veterans Affairs Medical Center, Cleveland, $\mathrm{OH}$
}

\begin{abstract}
We used a three-dimensional biomechanical model of human standing to test the feasibility of feed-forward control systems that vary stimulation to paralyzed muscles based on the user's posture and desire to effect a postural change. The controllers examined were (1) constant baseline stimulation, which represented muscle activation required to maintain erect standing, and (2) posture follower, which varied muscle activation as a function of the location of the projection of whole-body center of mass on the base of support. Posture-dependent control of stimulation demonstrated significant benefits over open-loop stimulation. Posture follower reduced upper-limb (UL) effort by an average of $50 \%$ compared with UL effort alone and by an average of $34 \%$ compared with baseline stimulation. On the other hand, reduction in UL effort was an average of $32 \%$ when using baseline stimulation. Compared with using UL effort alone, both controllers result in more than a $50 \%$ reduction in effort. The results of this study indicate that control systems that facilitate user-driven, task-dependent postures can be more effective and efficient than conventional open-loop stimulation. Also, they obviate the need for complicated posture-setting devices such as switches and joysticks. Functional implications include the potential to expand reachable workspace and better preparation for anticipated disturbances that could challenge balance over existing neuroprostheses for standing.
\end{abstract}

Key words: biomechanical model, FNS, functional neuromuscular stimulation, human standing, musculoskeletal modeling, neuroprosthesis, posture shifting, reaching, spinal cord injury, standing balance.

\section{INTRODUCTION}

Motor system neuroprostheses utilizing functional neuromuscular stimulation (FNS) can provide a means to improve the overall health and functional independence of individuals with paralysis resulting from spinal cord injury (SCI). Advanced FNS systems have been designed and deployed in several settings to aid individuals with SCI to stand erect from their wheelchairs [1] and to modulate stimulation levels according to sensor-based feedback to maintain balance against postural perturbations [2-3]. The use of such systems can have both physiological and functional benefits ranging from increased blood flow and muscle mass and reduced incidence of pressure sores [4 5] to the ability to access and interact with objects in the environment in ways that are difficult or impossible while

\footnotetext{
Abbreviations: $3 \mathrm{D}=$ three-dimensional, $\mathrm{AMAG} 1=$ adductor magnus, $\mathrm{AP}=$ anterior-posterior, $\mathrm{BoS}=$ base of support, $\mathrm{CoM}=$ center of mass, DOF $=$ degree of freedom, ESPINAE = erector spinae, FNS = functional neuromuscular stimulation, GMAX1 = gluteus maximus, GMED1 = gluteus medius, LL = lower limb, MEDGAS $=$ medial gastrocnemiums, $\mathrm{ML}=$ medial-lateral, $\mathrm{PD}=$ proportional derivative, $\mathrm{SCI}=$ spinal cord injury, SEMIMEM = semimembranosus, $\mathrm{UL}=$ upper limb, VASLAT = vastus lateralis. *Address all correspondence to Musa L. Audu, PhD; Motion Study Laboratory C15, Cleveland VA Medical Center, Cleveland, OH 44106; 216-791-3800, ext 3821; fax: 216707-6489. Email: mxa93@case.edu
}

http://dx.doi.org/10.1682/JRRD.2013.06.0150 
sitting. Current neuroprostheses for standing after SCI prevent collapse by stiffening the lower limbs (LLs) through constant continuous stimulation of the knee, hip, and trunk extensors. One drawback of this implementation is that it does not allow for modulation of the stimulation in order to enable the user to assume postures other than remaining rigidly erect at the nominal standing position. Any postural shifts required by the user to reach objects within or outside the immediate workspace or to prepare for activities or expected disturbances must be achieved by using the arms to pull or push against an assistive device such as a walker. Since constant stimulation patterns are customized to support a specific standing posture (i.e., erect neutral stance), these patterns will be suboptimal and will not aid in changing posture away from neutral. This is because leaning away from neutral standing may require more muscle effort to support the body against the constant pull of gravity. Assuming the whole-body center of mass (CoM) was located $1 \mathrm{~m}$ above ground, a $10^{\circ}$ forward lean about the ankle joints would result in an increase in ankle torque of more than $120 \mathrm{~N}-\mathrm{m}$ for a $75 \mathrm{~kg}$ individual. If additional muscle effort were not recruited to absorb this torque, it would have to be provided by the upper limbs (ULs).

The ability to alter standing posture has several potential advantages. By changing the posture from one position to another, muscles of one side of the body could be rested to mitigate fatigue and thus prolong overall standing times. It would also be beneficial for users of standing neuroprostheses to shift their postures in preparation for tasks such as lifting and moving objects to and from shelves. In all such maneuvers, the location of the projection of the overall body CoM on the base of support (BoS) will have to be changed smoothly and continuously. Previous work has determined that the ability to execute maneuvers such as shifting weight from side to side or front to back would be essential to enable users of future FNS systems to adjust their postures in executing a wide variety of everyday tasks [6].

Studies of bipedal standing with neuroprostheses are difficult to realize experimentally in real-time because of the limitations of existing FNS systems, which include activation of only a few paralyzed muscles at a time. Elapsed standing times with conventional FNS systems are also relatively short, typically $10 \mathrm{~min}$, because of the fatigue induced by continuously activating postural muscles [7]. For these reasons, the most effective means for achieving a wide variety of postures and standing maneuvers are best explored in computer simulations that capture as many characteristics of the physical system as possible. Several musculoskeletal models have been proposed and used for the study of standing balance with FNS [8-10]. A major advantage of a model-based approach is the ability to examine a large number of scenarios without having to resort to difficult and potentially impractical human experimentation. Recent simulation studies have shown that it is possible to hold the body statically at different postures in a bipedal stance using muscles that have been compromised due to paralysis [11-12].

Other investigators have utilized sophisticated biomechanical models to examine the potential reduction in degrees of freedom (DOFs) that could be achieved by the closed-kinematic chain that defines bipedal standing [13]. From these studies, it is clear that it is not necessary to activate all joints in a bipedal standing maneuver. Only a minimal number commensurate with the total DOFs of the system need to be actively controlled, thus potentially reducing the number of stimulus channels required in a standing neuroprosthesis. We have developed a comprehensive and realistic three-dimensional (3D) musculoskeletal model that takes these and other biomechanical factors into account [14].

We hypothesize that standing performance can be improved in terms of reduced use of the ULs for support by developing a control system that modulates stimulation based on current user posture and the desire for a postural shift. The goal of this study was to test, in simulation, the feasibility of two feed-forward control systems that would enhance the ability of neuroprosthesis recipients to change their standing postures. In the current work, the UL forces were envisaged as the major input to the system while the excitation of the muscles were altered only to assist with the intended movements generated by the user pulling or pushing on a walker with his or her arms. In this way, stimulation "follows" the new CoM locations acquired as a consequence of applying the UL forces rather than resisting movement of the CoM, as in the case of conventional continuous stimulation for standing. Due to the complex nature of the task and limitations with working with live subjects, this initial study was performed using a 3D biomechanical standing human model [15]. To simulate physiologically useful postural changes, the model pose was changed by specifying the UL forces required to place the overall body $\mathrm{CoM}$ in various locations within the $\mathrm{BoS}$, as defined by the two feet in a nominal standing posture. 


\section{METHODS}

\section{Model Description}

A closed-chain model of the human legs and trunk developed in SIMM (Musculographics Inc; Santa Rosa, California) was used in this study. The model had 14 DOFs and was actuated by 16 muscles that were previously determined to be an optimal set for standing after SCI [12]. The muscles were Hill-type muscle models and had their maximal forces scaled to reflect the actual forces expected to be available in subjects with SCI [16-17]. The 14 DOFs were trunk pitch; trunk lateral bending (roll) angles; and bilateral (right and left) subtalar, ankle, knee, hip flexion and extension, hip adduction and abduction, and hip internal and external rotation angles. The muscles actuating the model were right medial gastrocnemius (MEDGAS), tibialis anterior, vastus lateralis (VASLAT), semimembranosus (SEMIMEM), adductor magnus (AMAG1), gluteus medius (GMED1), gluteus maximus (GMAX1), and erector spinae (ESPINAE). The left side muscle names were prepended with the letter "L." Point forces were applied to each of the model's shoulders to simulate interactions with an assistive device such as a walker. The magnitude and direction of the forces were set by a proportional-derivative (PD) controller that used changes in the shoulder positions as input. The mathematical description of the model (skeleton and muscles) takes the form of a set of ordinary differential equations that were integrated to determine the joint trajectories. The inputs to the model were the muscle forces, UL forces, and gravity; the outputs were joint trajectories. The segmental mass and inertia properties of the model were calculated according to anthropometric tables [18] based on an average healthy male (weight $85 \mathrm{~kg}$, height $1.72 \mathrm{~m}$ ). The details of the model development and its application to posture shifting maneuvers were given in Audu et al. [15].

\section{Posture Optimization}

A sequential quadratic programming optimization routine (SNOPT) [19] was used to find a set of postures that would allow the body's CoM to be located across a range of positions while maximizing the height of the vertical component of the CoM and minimizing the total change in joint angles. The objective function for this optimization was defined as (Equation (1)) -

$$
J(\overrightarrow{\mathrm{q}})=\sum_{\mathrm{i}=1}^{\mathrm{n}} \Delta \mathrm{q}_{\mathrm{i}}^{2}+\frac{1}{\left(\mathrm{CoM}_{\mathrm{IS}}\right)^{2}} .
$$

where $\vec{q}=$ the vector of the generalized coordinates that were adjusted to change the location of the projection of the CoM on the BoS; $\Delta q_{i}=$ the change in joint angles from the neutral posture, which consisted of an erect standing posture with the feet placed shoulder-width apart; and $\mathrm{CoM}_{\mathrm{IS}}=$ the vertical (inferior-superior) component of the CoM. J( $\vec{q})$ was to be minimized subject to two equality constraints (Equation (2)):

$$
\begin{aligned}
& \mathrm{g}_{1}(\overrightarrow{\mathrm{q}})=\mathrm{CoM}_{\mathrm{AP}}^{\text {actual }}-\mathrm{CoM}_{\mathrm{AP}}^{\text {desired }}=0 \\
& \mathrm{~g}_{2}(\overrightarrow{\mathrm{q}})=\mathrm{CoM}_{\mathrm{ML}}^{\text {actual }}-\mathrm{CoM}_{\mathrm{ML}}^{\text {desired }}=0
\end{aligned},
$$

where $\mathrm{CoM}_{\mathrm{AP}}$ and $\mathrm{CoM}_{\mathrm{ML}}=$ the anterior-posterior (AP) and medial-lateral (ML) components of the CoM, respectively, with the superscripts denoting the "desired" and "actual" values. The second term in Equation (1) was added to maintain the body as erect as possible while ensuring that the joint angles appropriately changed to achieve the desired CoM position.

Optimal postures were determined for CoM locations ranging $10 \mathrm{~cm}$ left and right of neutral and $22 \mathrm{~cm}$ forward from neutral in $2 \mathrm{~cm}$ increments. The neutral posture located the CoM at $5.2 \mathrm{~cm}$ in the AP direction and $0.0 \mathrm{~cm}$ in the ML direction measured from the ground reference frame located at the midpoint between the calcaneus bones of the two feet. Figure 1 shows the CoM locations

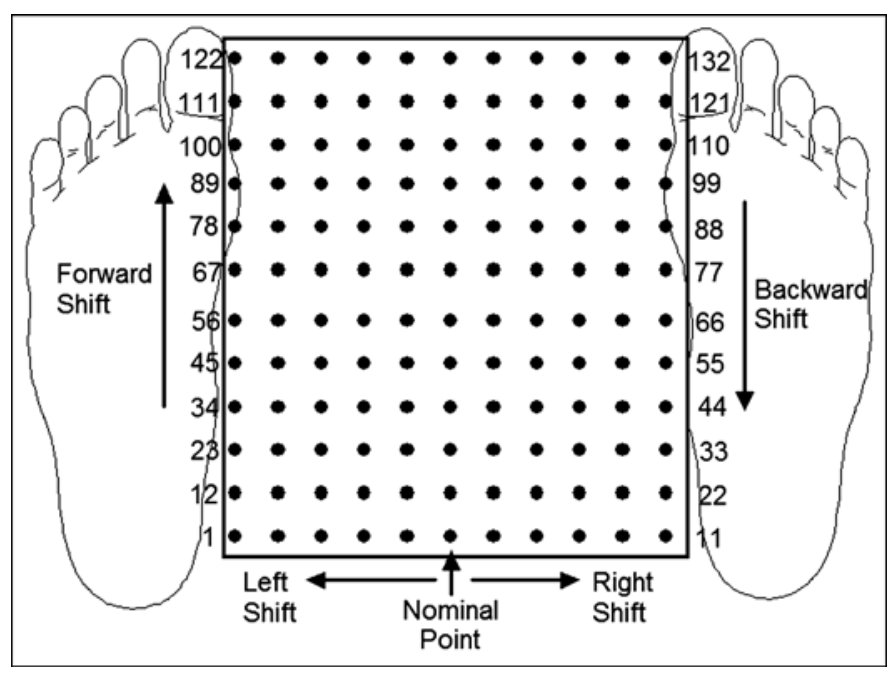

Figure 1.

Grid defined within boundaries of base of support defined by two feet of person in nominal standing posture. Center of mass is located statically at each of 132 grid points. Movements (shifts) are defined as those from one grid point to neighboring point. 
defined in a grid on the estimated BoS that covered the area between the two feet for a typical normal human standing with feet placed shoulder-width apart [20]. The optimization routine was repeated 40 times for each prescribed CoM location, each time from a different randomly generated set of initial joint angles. This was done to find a global minimum in the highly nonlinear solution space. The best solutions $\left(\mathrm{g}_{1}, \mathrm{~g}_{2} \leq 1.0 \mathrm{e}^{-4}\right.$ in Equation (2) and $\mathrm{CoM}_{\mathrm{IS}} \geq 1.3 \mathrm{~m}$ in Equation (1)) were selected for each prescribed CoM location, and a second-order polynomial surface was fit to the results for each joint angle. An initial study with polynomials of different degrees resulted in the choice of the second-order polynomial because it was the lowest degree that provided the best fit with mean square error less than 1 percent. This ensured that an optimal posture, defined by a set of joint angles, for any given CoM location could be calculated. The equation for the polynomial was (Equation (3)) -

$p=a_{1}+\left(a_{2} \cdot x_{G}\right)+\left(a_{3} \cdot y_{G}\right)+\left(a_{4} \cdot x_{G}^{2}\right)+\left(a_{5} \cdot y_{G}^{2}\right)+\left(a_{6} \cdot x_{G} \cdot y_{G}\right)$

where $\mathrm{x}_{\mathrm{G}}$ and $\mathrm{y}_{\mathrm{G}}=$ the $\mathrm{AP}$ and $\mathrm{ML}$ coordinates of the $\mathrm{CoM}$ on the grid, respectively, and $\mathrm{a}_{1} \ldots \mathrm{a}_{6}=$ parameters of the quadratic polynomial that best fit the polynomial data. The elements of the vector $(p)$ on the left-hand side of the equation were the 14 DOFs defining the joint angles in the model. A nonlinear least squares method optimization (function "lsqcurvefit," MATLAB, MathWorks; Natick, Massachusetts) was used to determine the six parameters for each of the joint angles. With the parameters $a_{1} \ldots a_{6}$ known, the values of the angles for every choice of CoM location in the plane could be estimated. The polynomial defined in Equation (3) defined what was called the "CoM-joint angle map." The main motivation for setting up the CoM-joint angle map was to identify the optimal joint angles for any specified CoM location that could serve as inputs to an inverse dynamics algorithm to determine the joint moments required to maintain the posture at that desired location.

In practice, the CoM-joint angle map could also be used to determine the CoM given the joint angles. However, this would be difficult to implement because it may require a large number of sensors to capture all the relevant values. In actual implementation, the CoM could be readily determined using a small number of inertial sensors appropriately placed at strategic locations on the body [12].

\section{Posture Controller Design}

The goal of the posture controller was to set muscle activations appropriately to allow for postural changes, thus maintaining sufficient muscle activity to support the body against gravity. Two controller paradigms were explored: a baseline controller that maintained the same muscle activations at the optimum values required to maintain an erect static standing posture and a posture follower that continuously adjusted muscle activations based on the instantaneous location of the CoM. Both controllers utilized a feedforward command signal from the neuroprosthesis user to set the desired CoM location by using the ULs to exert forces on a support device such as a walker or countertop. Figure 2 depicts the control setup.

The posture follower design employed a map between CoM position and muscle activation values to drive its actions. Inverse dynamic simulations were performed with the model CoM placed in each of the grid points in Figure 1 to determine the joint moments $T_{j}^{d}$ at each of the $(j=1 \ldots 14)$ DOFs required to statically maintain the posture at that grid point. The inputs to the inverse dynamics calculator were the joint angles read from the CoM-joint angles map. Once the joint moments were determined, a muscle force-sharing optimization problem was set up to determine the activations that would generate those moments. The objective function and constraints for that

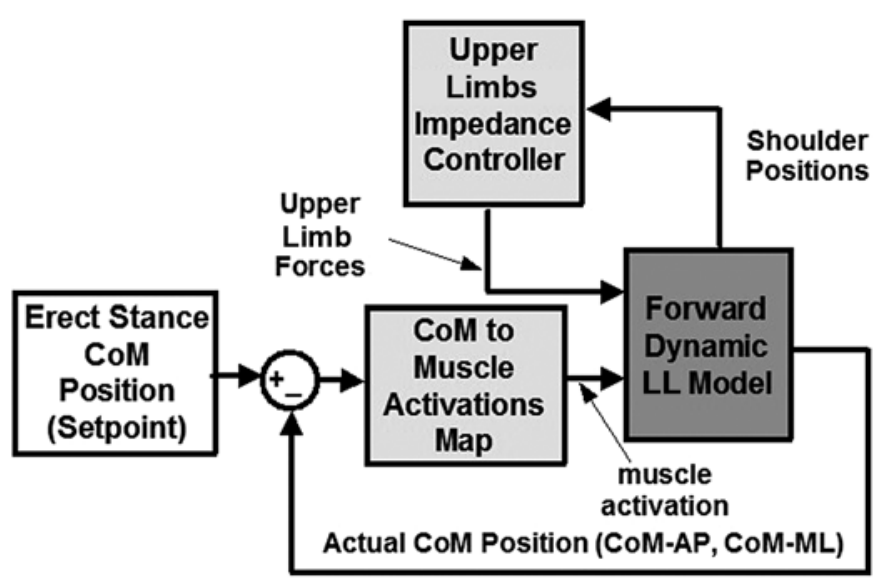

Figure 2.

Posture follower elements influencing center of mass (CoM) transitions from one location to another within base of support. User changes CoM location by using upper limbs to exert forces on support device. Muscle activations are changed to aid desired shift. $A P=$ anterior-posterior, $L L=$ lower $\operatorname{limb}, M L=$ medial-lateral. 
optimization take the following form typically used for similar optimization problems (Equations (4)-(5)) [21]:

$$
\begin{gathered}
J(a)=\sqrt{\sum_{i=1}^{n m}\left(\frac{F_{i}}{A_{i}}\right)^{2}} \quad i=1, \ldots, n m \text { and } \\
g_{j}=\left(T_{j}-T_{j}^{d}\right)=0 \quad j=1, \ldots, d f,
\end{gathered}
$$

where $\mathrm{J}=$ objective function, $a=$ vector of muscle activations, $n m=$ number of muscles, $F_{i}=$ force output from muscle $i, \mathrm{~A}_{\mathrm{i}}=$ physiologic cross-sectional area of muscle $i, d f=$ number of controlled DOFs, and $\mathrm{T}_{\mathrm{j}}=$ instantaneous torque produced at DOF $j$ by the muscles crossing it.

The mapping for the posture follower was developed by fitting a third-order polynomial surface to the required muscle activation values for each muscle against the location of the CoM within the BoS. The equation for the polynomial was given by (Equation (6)):

$$
\begin{aligned}
p_{s}=a_{1}+ & \left(a_{2} \cdot x_{G}\right)+\left(a_{3} \cdot y_{G}\right)+\left(a_{4} \cdot x_{G}^{2}\right)+\left(a_{5} \cdot y_{G}^{2}\right)+ \\
& \left(a_{6} \cdot x_{G} \cdot y_{G}\right)+\left(a_{7} \cdot x_{G}^{3}\right)+\left(a_{8} \cdot y_{G}^{3}\right)+ \\
& \left(a_{9} \cdot x_{G}^{2} \cdot y_{G}\right)+\left(a_{10} \cdot x_{G} \cdot y_{G}^{2}\right),
\end{aligned}
$$

where $\mathrm{x}_{\mathrm{G}}$ and $\mathrm{y}_{\mathrm{G}}=\mathrm{AP}$ and ML locations of the CoM grid point, respectively, and $\mathrm{a}_{1} \ldots \mathrm{a}_{10}=$ coefficient parameters of the third-order polynomial that fit the values of the static muscle activations defined by the quantity $\mathrm{p}_{\mathrm{s}}$ on the lefthand side of the equation. A nonlinear least squares method optimization was used to determine the 10 parameters for each of the static muscle activations. With this "static muscle activation map," the instantaneous values of the muscle activations required to maintain the model in any given posture within the BoS could be determined and applied continuously as the body moved from one CoM location to another.

\section{Upper-Limb Contribution}

Prior to doing forward simulations to test the controllers, the effect of the ULs on the dynamics of the movements was considered. In several studies, force production in the ULs was modeled as an impedance control phenomenon typically represented by an equation of form (Equation (7)) [22-24]:

$$
\mathrm{M} \frac{\mathrm{d}^{2}}{\mathrm{dt}^{2}} \delta \mathrm{X}(\mathrm{t})+\mathrm{B} \frac{\mathrm{d}}{\mathrm{dt}} \delta \mathrm{X}(\mathrm{t})+\mathrm{K} \delta \mathrm{X}(\mathrm{t})=\delta \mathrm{F}(\mathrm{t}),
$$

where $\mathrm{M}, \mathrm{B}$, and $\mathrm{K}=$ generalized inertia, damping, and stiffness factors, respectively, of the arm; $\delta \mathrm{X}(\mathrm{t})=$ change in hand position; and $\delta \mathrm{F}(\mathrm{t})=$ change in applied force at the hand. In supported standing, the maneuvers may involve slow movements characterized by negligible acceleration of the shoulders and, therefore, constant velocities. At such steady-state conditions, Equation (7) reduces to (Equation (8)) [25] -

$$
\mathrm{B} \frac{\mathrm{d}}{\mathrm{dt}} \delta \mathrm{X}(\mathrm{t})+\mathrm{K} \delta \mathrm{X}(\mathrm{t})=\delta \mathrm{F}(\mathrm{t}) \text {. }
$$

A fundamental assumption in our study was that since, in human standing, the hands were fixed in space (in contact with a static support device), the same equation as Equation (8) would model the impedance of the arm in force production such that shoulder movements in space $\delta \mathrm{X}(\mathrm{t})$ were accompanied by a force $\delta \mathrm{F}(\mathrm{t})$ at the shoulders. As a first approximation, therefore, interactions of the user with a support device were represented by the forces exerted at the shoulders as determined by a PD controller acting on changes in the shoulder positions [26-27]. At every instant of time during the simulation, the UL effort applied at the shoulders was computed via (Equation (9)) -

$$
\mathrm{UE}^{\mathrm{m}}=\sum_{\mathrm{i}=1}^{3} \mathrm{P}_{\mathrm{i}}^{\mathrm{m}}+\sum_{\mathrm{i}=1}^{3} \mathrm{D}_{\mathrm{i}}^{\mathrm{m}}
$$

where the first term was the proportional term defined as (Equations (10)-(11)) -

$$
\begin{array}{r}
P_{i}^{m}=K_{P i}^{m} \cdot e_{i}^{m} \text { and } \\
e_{i}^{m}=\left(\operatorname{IPS}_{i}^{m}-C^{m} S_{i}^{m}\right),
\end{array}
$$

and the second term in Equation (9) was the derivative term defined as (Equations (12)-(13)) -

$$
\begin{gathered}
D_{i}^{m}\left(t_{k}\right)=\frac{T_{D}}{T_{D}+N \Delta t}\left[D_{i}^{m}\left(t_{k-1}\right)-K_{P_{i}}{ }^{m} e_{i}^{m}\right] \text { and } \\
T_{D}=K_{D i}^{m} / K_{P i}^{m}
\end{gathered}
$$

where $\mathrm{UE}_{\mathrm{i}}^{\mathrm{m}}$ represents the UL forces applied at the right $(m=\mathrm{R})$ or the left $(m=\mathrm{L})$ shoulders along the fore-aft $(i=$ $1)$, ML $(i=2)$, and inferior-superior $(i=3)$ directions; $\mathrm{K}_{\mathrm{Pi}}^{\mathrm{m}}$ and $\mathrm{K}_{\mathrm{Di}}^{\mathrm{m}}$ are the proportional and derivative 
gains (similar meanings apply for $i$ and $m$ as for $\mathrm{UE}_{\mathrm{i}}^{\mathrm{m}}$ ). $\mathrm{IPS}_{i}^{\mathrm{m}}$ and $\mathrm{CPS}_{i}^{\mathrm{m}}$ represent the instantaneous and commanded positions of the shoulders, respectively. The positions of the left and right shoulders were computed from the model using the orientations of the body segments. The commanded positions of the shoulders were those computed with the body in the erect nominal standing position. Equation (12) represents a filtered derivative term using a first-order filter where $\mathrm{N}$ is a constant between 8 and 16 [28]; $\mathrm{T}_{\mathrm{D}}$ is the derivative time constant; and $\Delta \mathrm{t}$ is the controller update step size, set at $0.005 \mathrm{~s}$ for all simulations. Altogether, there were six proportional and six derivative gains to be determined before the PD controller was applied in simulation. An optimization algorithm where the overall body $\mathrm{CoM}$ was required to track a set of specified trajectories involving shifts in the AP, ML, and diagonal directions was used to determine the gains. The trajectories of the CoM were chosen arbitrarily to represent movements that would imitate actual shifts in body pose such as would be required to retrieve objects in the space around a standing human. They involved shifts in projections of the CoM from the neutral posture to a forward, sideways, or diagonal location within the BoS and return to neutral. The PD gains were determined so as to minimize an objective function given by (Equation (14)) -

$$
\begin{aligned}
& \mathbf{J}\left(\mathrm{K}_{\mathrm{Pi}}^{\mathrm{m}}, \mathrm{K}_{\mathrm{Di}}^{\mathrm{m}}\right)= \sum_{\mathrm{AP}, \mathrm{ML}, \text { diag }}\left[\sum_{\mathrm{i}=1}^{3}\left(\mathrm{CoM}_{\mathrm{i}}^{\text {actual }}-\mathrm{CoM}_{\mathrm{i}}^{\text {commanded }}\right)^{2}+\right. \\
&\left.\mathrm{W} \cdot \sum_{\mathrm{i}=1}^{3}\left(\mathrm{UE}_{\mathrm{i}}^{\mathrm{m}}\right)^{2}\right]
\end{aligned}
$$

where $\mathrm{CoM}_{\mathrm{i}}^{\text {actual }}$ and $\mathrm{CoM}_{\mathrm{i}}^{\text {commanded }}=$ actual and commanded positions of the body CoM along the AP, ML, or diagonal directions. $\mathrm{W}$ is a weighting parameter that weighs the tracking error relative to the UL effort during a movement. $\mathrm{W}$ was determined such that at the nominal standing posture with baseline stimulation, the two terms in square brackets in Equation (14) were about equal. That value was used throughout the rest of the optimization to scale the second term relative to the first.

In a forward simulation, the combined forces acting on the system, in addition to gravity, were (1) the UL effort representing the interaction with the walker and (2) muscle forces generated via constant baseline muscle activations or via variable muscle activations applied to the muscles as function of the instantaneous location of the projected whole-body CoM onto the BoS.
The impedance parameters (PD gains) for the UL contribution were determined as part of a series of dynamic simulations involving the five specific shifts in the CoM as depicted in Figure $\mathbf{3}$ for each of the three conditions: UL effort only, baseline activation, and posture follower. In every optimization step, the term in square brackets in Equation (14) was computed for each of the five movements, and the final objective was the sum of the five outcomes. After the gains for each of the three conditions were determined, their mean values were computed and used in the same optimization but with the objective function being the sum of the three objectives. To alleviate the computational burden, all optimization runs were done in a supercomputer cluster consisting of two 32-processor nodes running the parallel optimization software APPSPACK (Asynchronous Parallel Pattern Search Package) developed at the Sandia National Laboratories (Albuquerque, New Mexico) [29]. Thereafter, the performance of each control paradigm was tested by doing similar forward dynamic simulations of posture shifts that moved the whole-body CoM from the nominal position to five different targets individually in the transverse plane along straight-line trajectories. The targets were located in a neutral position, $10 \mathrm{~cm}$ to the left and right of neutral, $10 \mathrm{~cm}$ forward from neutral, and $10 \mathrm{~cm}$ at the left-forward and right-forward diagonal locations (Figure 3). Each simulation was $30 \mathrm{~s}$ in duration and consisted of an initial stabilization period of $5 \mathrm{~s}$, a movement from one target to an adjacent target (up ramp) with a duration of $6 \mathrm{~s}$, a dwell at

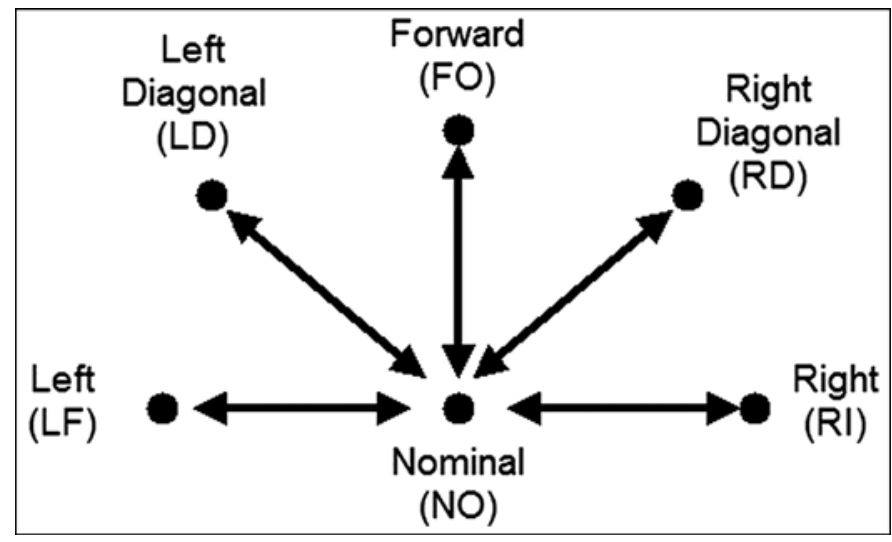

Figure 3.

Target locations for forward simulations with baseline stimulation and with posture follower. All movements commence from nominal posture. 
the target position for $8 \mathrm{~s}$, and a return to the initial target (down ramp) in $6 \mathrm{~s}$ with another stabilization period of $5 \mathrm{~s}$. For comparison purposes, the simulations were repeated with no muscles active. Only the UL forces were used to execute the various movements, although reaching in these cases would not be possible since both limbs would always be required for support and balance.

\section{Testing Controller Performance}

The simulation procedures for testing controller performance were the same as those used to determine the PD gains in the previous section. These simulations were carried out to test the two stimulation conditions: (1) "open-loop control" in which constant, optimal (or baseline) activations were applied and the ULs generated the movement and (2) "posture follower control" in which stimulation was modulated based on the current location of the CoM. The UL effort required to generate the movement was used as a metric of system performance. The differences between the UL efforts used when the controllers were active against the situation when the UL forces acted alone with no muscles active were assessed using (Equation (15)) -

$$
\% \text { difference }=\frac{E_{\mathrm{UE}}-\mathrm{E}_{\mathrm{CO}}}{\mathrm{E}_{\mathrm{UE}}} \cdot 100,
$$

where $\mathrm{E}_{\mathrm{UE}}=$ mean effort without stimulation and only the ULs were active, and $\mathrm{E}_{\mathrm{CO}}=$ mean effort when one of the two controllers (baseline stimulation or posture follower) was active. A similar approach was used to determine the performance of the posture follower relative to the conventional clinical case of standing with continuous constant stimulation. The mean efforts were computed as the areas under the curve of the UL forces over the time interval $0.25 \mathrm{~s} \leq t \leq 30 \mathrm{~s}$. The period up to $t=0.25 \mathrm{~s}$ was taken as the stabilization period when the body was adjusting and thus was ignored in the integration.

\section{RESULTS}

\section{Posture Optimization}

Figure 4 shows 3D plots of the profiles of each of the 14 joint angles versus the AP and ML locations of each grid point. The asterisks show the actual data points while the continuous colored mat shows the fitted map. The results of the optimization routine indicate that a combination of ankle, hip, and trunk angle changes contributed mainly to movement of the CoM in the sagittal (AP direction) plane. The knees and rotation of the hips were fairly constant for all changes in CoM. There was no apparent contribution from hip adduction and trunk bend when the CoM moved forward or backward. For postures with the CoM shifted in the coronal (ML direction) plane, a combination of subtalar angle, hip abduction and adduction, and trunk bend were required. Trunk movement contributed most to the optimal maneuvers followed by subtalar motion, implying that trunk bend or roll and subtalar angles are the preferred methods for achieving change of $\mathrm{CoM}$ in the ML direction. Hip abduction and adduction were the least important contributor, and the remaining joint angles remained relatively constant as the CoM position was varied in the coronal plane.

\section{Upper-Limb Impedance Parameters}

The Table shows the values of the gains obtained using Equation (14). These parameters are applicable as UL impedance values for all movement directions and for all controller types. The mean UL proportional gains were largest in the inferior-superior direction (mean $5,020 \mathrm{~N} / \mathrm{m}$ ). These are followed by those in the fore-aft direction (mean 3,170 N/m) and then those in the ML direction (mean $1,330 \mathrm{~N} / \mathrm{m}$ ). All derivative gains assume a mean value of $109 \mathrm{~N}-\mathrm{s} / \mathrm{m}$. The larger value of proportional gain in the inferior-superior direction may be attributable to the need to sustain the overall body weight in that direction, while the lower value in the ML directions may be due to the higher stability in that direction associated with the width of the BoS. Lower values for the derivative gain were important to avoid overshoots in the UL control forces despite utilizing filtered derivatives for all velocity inputs. A constant value of $1.0 \mathrm{e}^{-3}$ was found adequate for the weighting parameter (W) in Equation (14) for all cases.

\section{Posture Follower Map}

The posture follower relied on a series of maps between CoM locations in the transverse plane and the muscle activations required to (1) support the posture against gravity and (2) allow for generation of forward, backward, left, and right shifts in CoM location. Figure 5 shows plots of the activations of each of the 16 muscles versus the AP and ML locations of each grid point. These maps specifying the muscle activations required to support the body against gravity confirm the findings of previous reports that each of these muscles was important 
Right
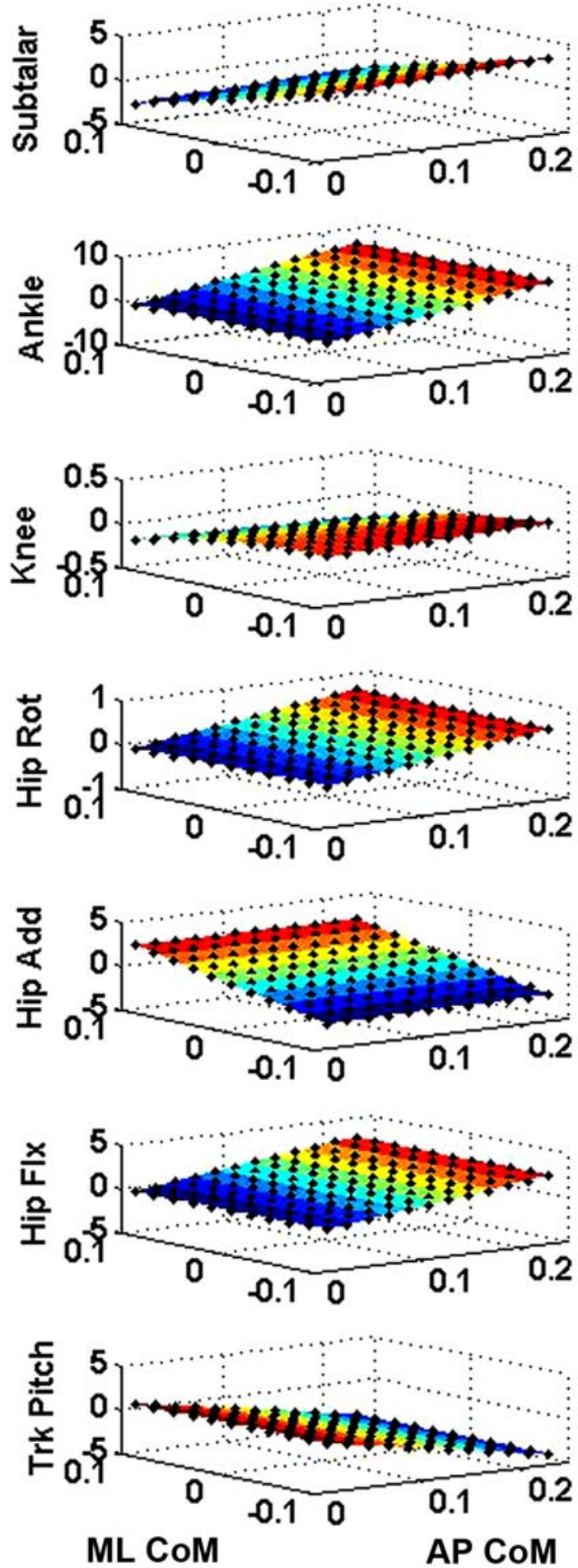

Left
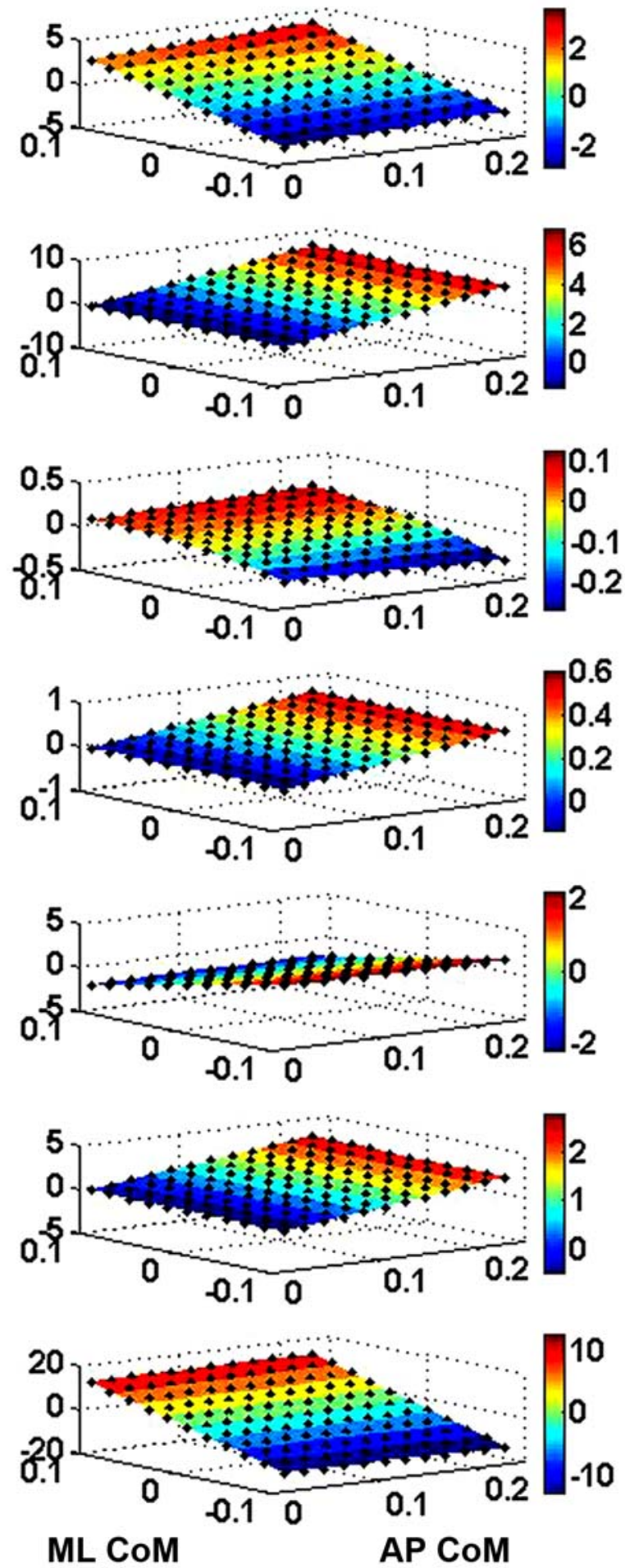

Figure 4.

Three-dimensional plots of 14 free degrees of freedom (joint angles) plotted against anterior-posterior (AP) and medial-lateral (ML) directions on base of support. Two bottom plots represent trunk pitch and trunk roll to left and right, respectively. *Actual data points (continuous colored mat shows fitted map). Add $=$ adduction, $\mathrm{CoM}=$ center of mass, Flx $=$ flexion, Rot $=$ rotation, Trk $=$ trunk. 
Table.

Gains for upper-limb proportional-derivative controller.

\begin{tabular}{lccc}
\hline Shoulder & Gain Type & $\begin{array}{c}\text { Movement } \\
\text { Direction }\end{array}$ & Value \\
\hline Right & Proportional $(\mathrm{N} / \mathrm{m})$ & $x$ & $3.17 \mathrm{E}+03$ \\
& & $y$ & $5.02 \mathrm{E}+03$ \\
& Derivative $(\mathrm{N}-\mathrm{s} / \mathrm{m})$ & $x$ & $1.33 \mathrm{E}+03$ \\
& & $y$ & $1.09 \mathrm{E}+02$ \\
& & $z$ & $1.09 \mathrm{E}+02$ \\
& & $x$ & $3.17 \mathrm{E}+03$ \\
Left & Proportional $(\mathrm{N} / \mathrm{m})$ & $y$ & $5.03 \mathrm{E}+03$ \\
& & $z$ & $1.33 \mathrm{E}+03$ \\
& & $x$ & $1.09 \mathrm{E}+02$ \\
& Derivative $(\mathrm{N}-\mathrm{s} / \mathrm{m})$ & $y$ & $1.09 \mathrm{E}+02$ \\
& & $z$ & $1.09 \mathrm{E}+02$ \\
\hline Note: $x=$ fore-aft, $y=$ inferior-superior, $z=$ medial-lateral. & \\
\hline
\end{tabular}

for standing in various static postures [12]. The muscles with the highest activations across all tested postures were those that are traditionally associated with postural support: ankle plantar flexors to stiffen the ankle, SEMIMEM and ESPINAE to maintain hip and trunk extension, and AMAG1 and GMED1 to stiffen the hips against further adduction and abduction in sideways leaning postures. The muscles with lower average activation are still important to maintain specific nonerect postures; however, activations of the GMAX1 and VASLAT were small and relatively unchanged across all postures.

\section{Controller Performance}

The performance of the controllers was tested in a series of simulated postural shifts with the task of moving the CoM between various targets along a straight-line trajectory. Postural shifts between every adjacent target were tested while the mean UL effort required to generate the movement, as well as the mean error between target and actual CoM position in the transverse plane, was optimized. Figure 6 shows actual and commanded CoM positions in the transverse plane to illustrate the shape of the trajectories and tracking errors for each system. Figure 7 depicts temporal histories of the CoM trajectories for the forward, leftward, and left diagonal shifts for the posture follower, while Figure 8 depicts the histories of the joint angles obtained by integrating the model differential equations for the case of the forward leaning maneuver for all three conditions (UL effort alone, base- line stimulation, and posture follower) against the desired angles as computed from the CoM-joint angle map. From Figure 8, it appears that forward shift maneuvers were accomplished primarily by changes in ankle angle, with most of the other joint angles remaining relatively constant. All controllers exhibited good tracking for the relevant CoM components with very small overshoot during the left diagonal movements when the UL effort was used alone and with open-loop baseline stimulation.

Figure 9 shows the UL effort histories applied during the specified maneuvers. The mean UL effort required to maintain a steady-state posture at the nominal erect posture was between 90 and $100 \mathrm{~N}$ when the UL effort alone was used, while it reduced to 40 to $50 \mathrm{~N}$ when any of the muscle controllers were activated. Stimulation greatly reduced UL effort even in steady-state standing when the CoM was kept at a fixed position.

Figure 10 summarizes the percentage difference in mean UL force exerted during the time interval $(2.5 \mathrm{~s} \leq t \leq$ $30 \mathrm{~s})$ for each of the five maneuvers shown in Figure 9. Posture follower performed better than constant baseline stimulation (about 34\% reduction in UL effort) mainly during the forward and diagonal movements. During the extreme right and left movements, the reduction was about 8 percent. On the other hand, constant (open-loop) stimulation performed only slightly better than UL effort alone for the forward and diagonal movements. The approximately 20 percent improvement over no stimulation for this controller was due to reduced UL effort during the steady-state periods. During the extreme left and right movements, both controllers performed much better (up to about 50\%) than UL effort alone. The two controllers performed equally well during these two maneuvers when compared with no stimulation, probably because only portions of the ESPINAE muscles were recruited for movements in the ML plane (Figure 5). The other most active muscles (MEDGAS and SEMIMEM) were mainly active in the AP plane alone.

\section{DISCUSSION}

The primary goal of this study was to determine whether we could apply posture-specific stimulation to improve the performance of neuroprostheses for standing. We defined performance using two metrics: (1) reducing the effort required from the user to maintain a posture and switch between postures and (2) improving the user's 

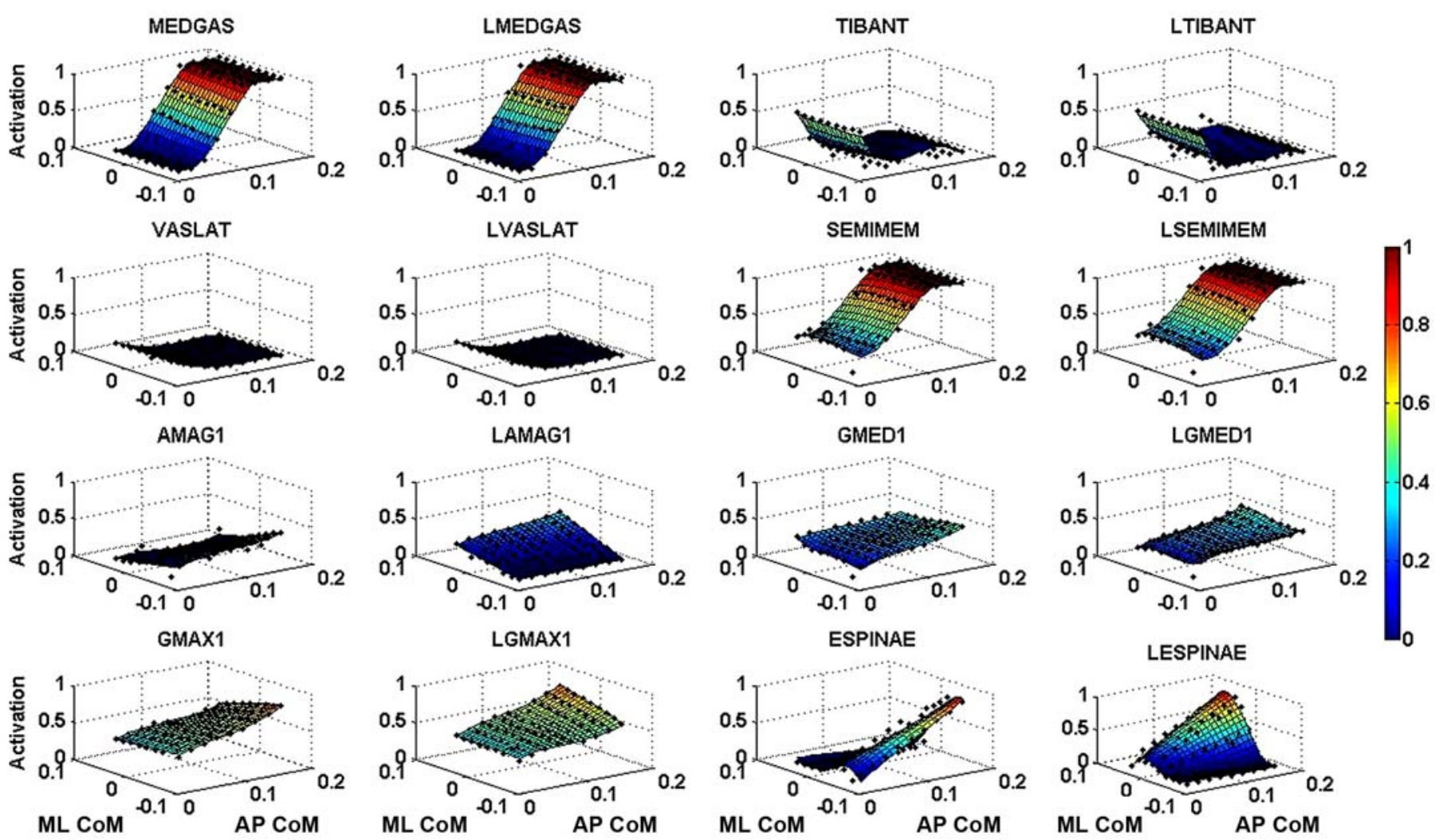

Figure 5.

Three-dimensional plots of 16 muscle activations for posture follower fitted to values of center of mass (CoM) grid locations on base of support. *Actual data points (continuous colored mat shows fitted map). AMAG1 = right adductor magnus, AP = anterior-posterior, ESPINAE = right erector spinae, GMAX1 = right gluteus maximus, GMED1 = right gluteus medius, LAMAG1 = left adductor magnus, LESPINAE = left erector spinae, LGMAX1 = left gluteus maximus, LGMED1 = left gluteus medius, LMEDGAS = left medial gastrocnemius, LSEMIMEM = left semimembranosus, LTIBANT = left tibialis anterior, LVASLAT = left vastus lateralis, MEDGAS = right medial gastrocnemius, $\mathrm{ML}=$ medial-lateral, SEMIMEM = right semimembranosus, TIBANT = right tibialis anterior, VASLAT = right vastus lateralis.

ability to maintain and track particular postures as defined by target CoM locations. Using a feed-forward control structure, the average UL effort was reduced by over 20 to 50 percent during postural shifts over using UL effort alone. CoM target tracking was excellent using all controller types with only a little overshoot during one of the maneuvers when the UL effort was used alone and when open-loop stimulation was used.

We tested two control structures: baseline open-loop stimulation and the posture follower. The baseline stimulation control structure was the simpler of the two and represented the conventional application of constant stimulation to all muscles. The posture follower varied muscle activation based on the location of the wholebody CoM in the transverse plane. This controller would allow optimal stimulation to be applied as the user moved between postures using his or her arms to drive the movement, in essence using the body itself as a joystick-like command input. The average UL effort was reduced by over 50 percent during postural shifts with the posture follower for all five maneuvers, over the 20 to 45 percent reduction with conventional open-loop continuous stimulation. The performance of continuous baseline stimulation was comparable with the posture follower only for the extreme leftward and rightward shifts. For the other three movements, the posture follower outperformed baseline stimulation by achieving up to 34 percent further reduction in UL effort. As illustrated in Figure 9, any form of LL stimulation reduced the UL effort required to support the body in the nominal posture and move the 

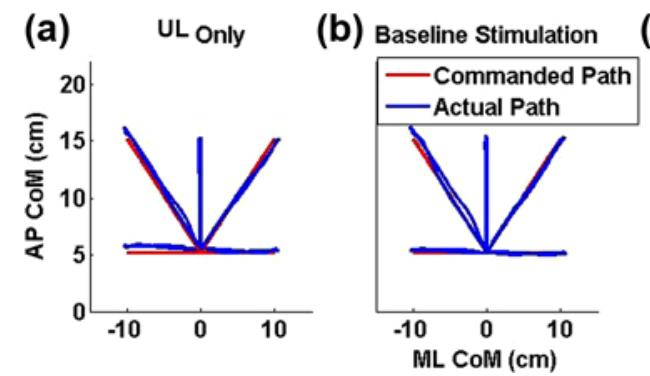

(c) Posture follower

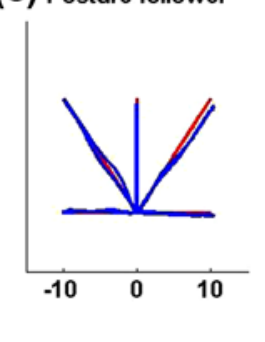

Figure 6.

Actual and commanded center of mass (CoM) paths for case of applying (a) upper-limb (UL) effort only and two controller conditions of (b) baseline constant stimulation and (c) posture follower for five specified path trajectories depicted in Figure 3. $\mathrm{AP}=$ anterior-posterior, $\mathrm{ML}=$ medial-lateral.

CoM to new targets by more than half that required by using the ULs alone without stimulation. In reality, users of standing neuroprostheses in particular and individuals with SCI in general rarely stand to reach objects by using only their ULs and instead rely on stimulation or external orthoses, respectively.

The low UL effort required to shift between postures and the high precision of CoM target tracking while using the posture follower means that any additional control structure could only offer minor benefits at the cost of system complexity and need for additional control inputs. These findings indicate that it would be best to focus on transitioning the posture follower into a realworld system instead of adding the unnecessary complications that more sophisticated control structures such as joystick control, etc., would bring.

Our previous studies investigated use of $\mathrm{CoM}$ for feedback control based on 3D acceleration of CoM for the express purpose of disturbance rejection about the nominal posture setpoint $[2-3,26]$. Stimulation levels were modulated to respond to unanticipated, dynamic changes occurring only while standing at that nominal position. However, constant baseline stimulation was utilized for vertical support while volitional user effort along with the actions of the controller maintained it at the nominal steady state value. In the current study, we investigated a potential to improve the clinical utility and functionality of standing by exploring an advanced control system that allows efficient shifts of the nominal posture itself. Specifically, this study explored modulating stimulation to more efficiently shift to other setpoint
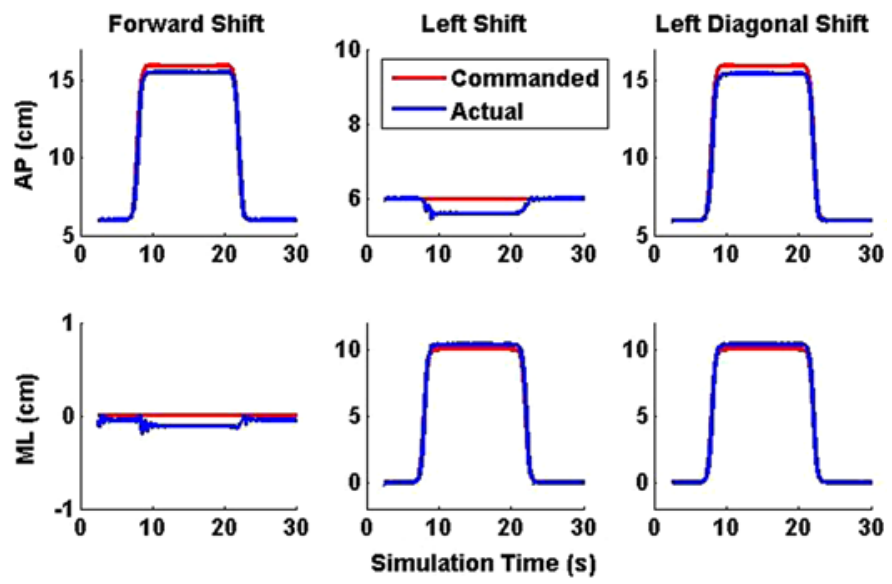

Figure 7.

Typical center of mass (CoM) trajectories (actual and commanded) for case of posture follower active. Top row: Changes in anterior-posterior (AP) CoM. Bottom row: Changes in mediallateral $(\mathrm{ML})$ CoM.

positions in concert with user volition. As such, CoM position was the process variable of interest. This variable can be estimated online from joint position measured by any number of methods, including segment accelerations that would avoid implementing awkward body-worn sensors that cross the hips, knees, and ankles. The posture follower approach, therefore, is compatible with the purely dynamic feedback provided by accelerometers located on the trunk and pelvis to estimate CoM accelerations for disturbance rejection.

Regardless of the control structure that is chosen, the transition to laboratory demonstration and clinical implementation will be a challenge. The primary limitation of either control system is the use of the whole-body CoM as a surrogate for body posture. Two inherent assumptions were made in the development of these controllers: (1) we can enforce a desired set of postures so that there is an optimal 1-to-1 mapping between CoM position and posture, and (2) we can determine CoM position with sufficient precision to accurately drive the controller. The first assumption is important because the muscle activations will be optimized for a specific set of postures, and if the user deviates too far from these postures, then these activations will not be optimal and could be detrimental to stable standing. This issue will need to be investigated further, but if stimulation alone is insufficient to generate unique, optimal postures, then the appropriate orthoses could be added at the problematic joints for a direct and 

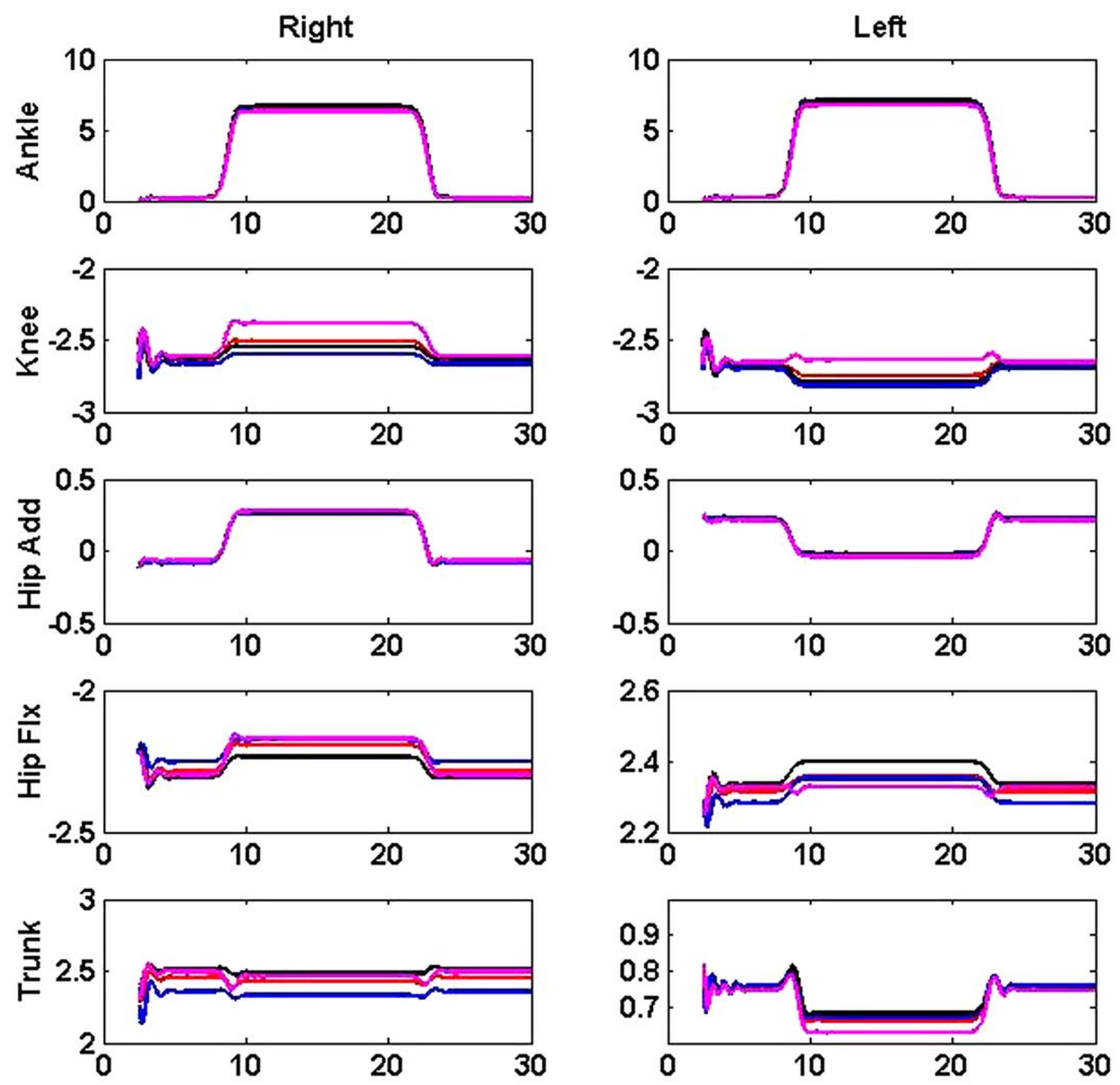

Simulation Time (s)

Figure 8.

Histories of joint angles obtained by integrating model differential equations for three types of loading conditions: upper-limb (UL) effort alone (blue), UL effort and baseline stimulation (black), and UL effort and posture follower (magenta). Commanded joint angles obtained from center of mass-joint angle map are shown in red. Add = adduction, Flx = flexion. 


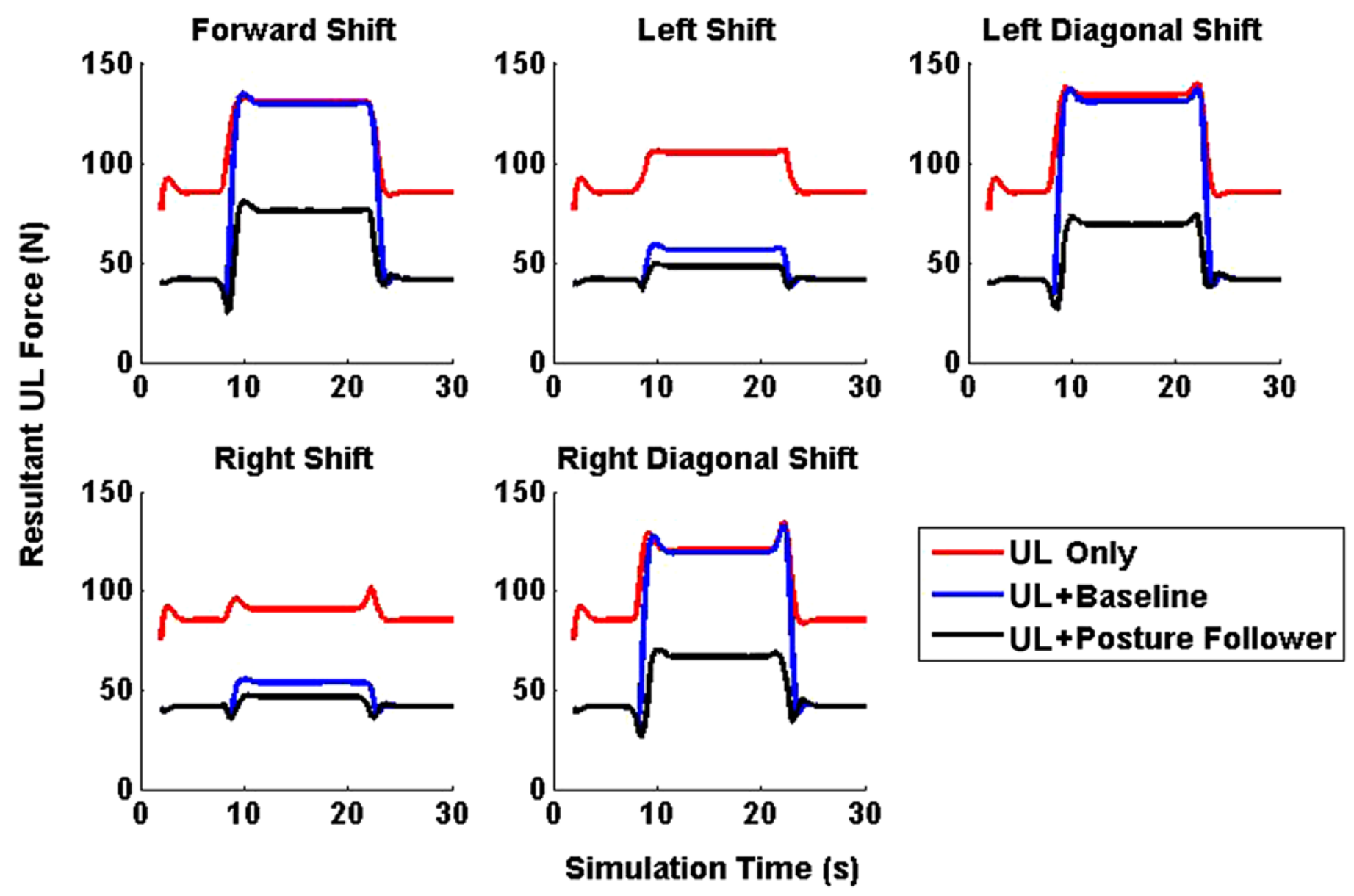

Figure 9.

Upper-limb (UL) effort (red line) exerted during different maneuvers shown in Figure 3 while applying two types of control elements: baseline stimulation (blue line) and posture follower (black line).

effective solution. The second issue is related to the relatively small variations in CoM location as the posture continually adapts and accommodates. Any system that tracks changes in CoM would need to be sensitive and robust in the presence of measurement noise. In the laboratory, optical motion-capture systems offer ample sensitivity to track very small changes in body position. Such systems are currently available for the home and in the community, although recent gaming technologies, such as the Nintendo Wii and Microsoft Kinect, provide hope that inexpensive, at-home motion-capture systems may eventually be possible. Currently, the best option would most likely be to use accelerometers or other orientation sensors placed at strategic locations on the trunk, pelvis, and limbs and to combine their signals in such a way as to give good estimates of whole-body CoM location and joint positions [26]. The simulation study described in Nataraj et al. determined that the typical noise and tracking errors reported for such sensors were within acceptable boundaries to still resolve postural positions with sufficient accuracy for effective feedback control of neuroprosthetic standing [30]. While the required precision of the sensors was not assessed in this study, the previous utilization of such sensors with our customized implantable stimulator for feedback control of neuroprosthetic standing suggests potential feasibility for live-subject implementation for setpoint control as well [2-3].

The model-based study presented here has some intrinsic limitations. First, the assumption that the UL contributes to the control of movement via a PD-type action is an oversimplification of the yet unknown behavior of the UL effort of a user under live conditions. It is 
JRRD, Volume 51, Number 3, 2014

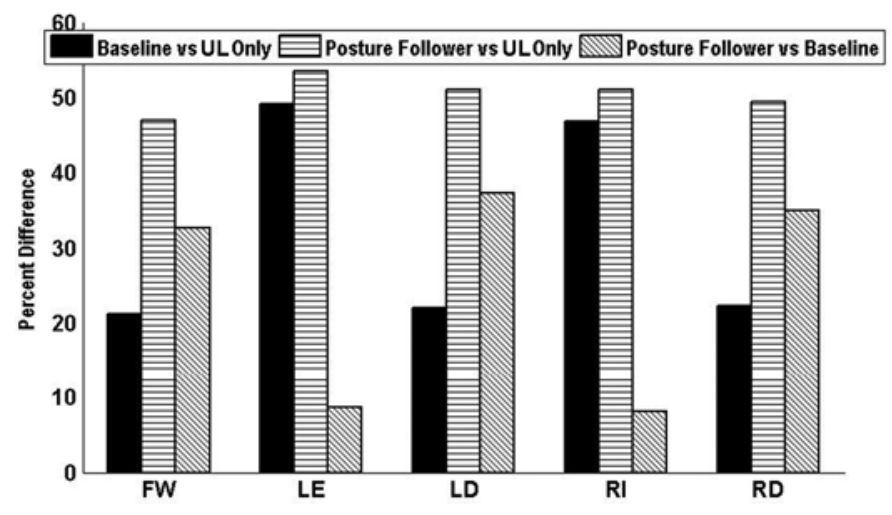

Figure 10.

Percent difference in mean upper-limb (UL) effort with baseline continuous open-loop stimulation and posture follower relative to UL effort alone and baseline continuous open-loop stimulation relative to posture follower calculated during forward (FW), left (LE), left diagonal (LD), right (RI), and right diagonal (RD) shifts shown in Figure 3.

unclear how best to validate that the ULs behave in the same manner as impedance control systems for hand manipulation. Further studies need to be carried out to validate the nature of UL contributions to movements in the AP and ML directions, especially when LL muscle action is compromised. Another limitation is that the maps were developed using static information only. The maps could be developed using the actual dynamic trajectories of the activations computed by undertaking a wide variety of dynamic maneuvers by changing the projected CoM from one random location to another. However, such maps would require more sophisticated and computationally expensive approaches, such as using dynamic neural networks. The potential benefits of such complex designs need to be further evaluated relative to their efficiency for clinical deployment. From Figure 9, we observed that the results did not seem to display an exact symmetry between the left and right and between the left diagonal and right diagonal UL forces. This also reflected in the percent difference in mean UL effort shown in Figure 10. The main reason for this lack of symmetry was because of the nature of the closed-chain constraint associated with the requirement to have the two feet be firmly placed on the ground throughout the simulations. In reality, only the right foot was fully constrained to the ground. The requirement for having the left foot also to be constrained fully to the ground could only be achieved via the satisfaction of an algebraic con- straint at every time step of the simulation. Thus, any model involving bipedal standing must solve a combined differential and algebraic set of equations [13,15]. Small errors in satisfying these algebraic constraint equations could cause the outputs between left and right sides to differ. This problem could be avoided by completely eliminating the closed-chain constraint equations from the model prior to any simulations. This approach would require enormous additional effort in the modeling of the system and is currently under study. Finally, the musculoskeletal model assumed there was no twist movement of the trunk. For many useful activities of daily living, control of trunk twist notably improves functionality. However, excessive axial rotation should be avoided for a neuroprosthesis user because this could compromise the stability attained while holding on to the support device.

This study sought to determine the feasibility of a posture-dependent muscle activation controller for a standing FNS system. Our results show that such a system could benefit a neuroprosthesis user by reducing the effort required to change postures while standing and allowing for finer control of posture. Both of these benefits would make reaching and performing activities while standing easier for a user of the standing system.

\section{CONCLUSIONS}

We have explored the potential of designing simple control structures that could be implemented in neuroprostheses to restore standing balance and allow individuals paralyzed by SCI to set task-specific and user-driven standing postures. By using smooth interpolating maps to determine which instantaneous muscle activations to apply in order to achieve movement maneuvers in the sagittal and coronal planes, we obviate the need for designing and deploying otherwise complex controllers for an inherently unstable and highly nonlinear biomechanical system. The results showed that advanced feedforward systems are feasible and can potentially outperform conventional open-loop baseline stimulation in terms of reduced UL effort. The application of such control structures will expand the capabilities of current FNS systems, which rely on constant baseline stimulation to maintain standing balance. 


\section{ACKNOWLEDGMENTS}

\section{Author Contributions:}

Study concept and design: R. J. Triolo, M. L. Audu, S. J. Gartman, R. Nataraj.

Computer simulations: M. L. Audu, S. J. Gartman.

Analysis and interpretation of results: R. J. Triolo, M. L. Audu, S. J. Gartman, R. Nataraj.

Preparing draft manuscript: S. J. Gartman, M. L. Audu. Critical revision of manuscript for important intellectual content: R. J. Triolo.

Study supervision: R. J. Triolo, M. L. Audu.

Financial Disclosures: The authors have declared that no competing interests exist.

Funding/Support: This material was based on work supported by the National Institute of Neurological Disorders and Stroke (grant R01NS040547).

Additional Contributions: Mr. Gartman is now with The Progressive Corporation, Mayfield Village, Ohio.

\section{REFERENCES}

1. Davis JA Jr, Triolo RJ, Uhlir JP, Bieri C, Rohde L, Lissy D, Kukke S. Preliminary performance of a surgically implanted neuroprosthesis for standing and transfers-where do we stand? J Rehabil Res Dev. 2001;38(6):609-17. [PMID:11767968]

2. Nataraj R, Audu ML, Triolo RJ. Center of mass acceleration feedback control of standing balance by functional neuromuscular stimulation against external postural perturbations. IEEE Trans Biomed Eng. 2013;60(1):10-19. [PMID:22987499] http://dx.doi.org/10.1109/TBME.2012.2218601

3. Nataraj R, Audu ML, Triolo RJ. Center of mass acceleration feedback control of functional neuromuscular stimulation for standing in presence of internal postural perturbations. J Rehabil Res Dev. 2012;49(6):889-911.

[PMID:23299260]

http://dx.doi.org/10.1682/JRRD.2011.07.0127

4. Chen $\mathrm{CF}$, Lien IN, Wu MC. Respiratory function in patients with spinal cord injuries: Effects of posture. Paraplegia. 1990;28(2):81-86. [PMID:2235026]

http://dx.doi.org/10.1038/sc.1990.10

5. Walter JS, Sacks J, Othman R, Rankin AZ, Nemchausky B, Chintam R, Wheeler JS. A database of self-reported secondary medical problems among VA spinal cord injury patients: Its role in clinical care and management. J Rehabil Res Dev. 2002;39(1):53-61. [PMID:11926327]

6. Abbas JJ, Gillette JC. Using electrical stimulation to control standing posture. IEEE Contr Syst. 2001;21(4):80-90. http://dx.doi.org/10.1109/37.939946
7. Triolo RJ, Bailey SN, Miller ME, Rohde LM, Anderson JS, Davis JA Jr, Abbas JJ, DiPonio LA, Forrest GP, Gater DR Jr, Yang LJ. Longitudinal performance of a surgically implanted neuroprosthesis for lower-extremity exercise, standing, and transfers after spinal cord injury. Arch Phys Med Rehabil. 2012;93(5):896-904. [PMID:22541312] http://dx.doi.org/10.1016/j.apmr.2012.01.001

8. Matjacić Z, Bajd T. Arm-free paraplegic standing-Part I: Control model synthesis and simulation. IEEE Trans Rehabil Eng. 1998;6(2):125-38. [PMID:9631320] http://dx.doi.org/10.1109/86.681178

9. Hung J, Gollee H, Jaime RP, Donaldson N. Design of feedback controllers for paraplegic standing. IEEE Proc Control Theory Appl. 2001;148(2):97-108.

10. Soetanto D, Kuo CY, Babic D. Stabilization of human standing posture using functional neuromuscular stimulation. J Biomech. 2001;34(12):1589-97. [PMID:11716861] http://dx.doi.org/10.1016/S0021-9290(01)00144-0

11. Heilman BP, Audu ML, Kirsch RF, Triolo RJ. Selection of an optimal muscle set for a 16-channel standing neuroprosthesis using a human musculoskeletal model. J Rehabil Res Dev. 2006;43(2):273-86. [PMID:16847793] http://dx.doi.org/10.1682/JRRD.2005.04.0072

12. Gartman SJ, Audu ML, Kirsch RF, Triolo RJ. Selection of optimal muscle set for 16-channel standing neuroprosthesis. J Rehabil Res Dev. 2008;45(7):1007-18. [PMID:19165690] http://dx.doi.org/10.1682/JRRD.2007.10.0164

13. Kim JY, Popovic MR, Mills JK. Dynamic modeling and torque estimation of FES-assisted arm-free standing for paraplegics. IEEE Trans Neural Syst Rehabil Eng. 2006; 14(1):46-54. [PMID:16562631]

14. Zhao W, Kirsch RF, Triolo RJ, Delp S. A bipedal, closedchain dynamic model of the human lower extremities and pelvis for simulation-based development of standing and mobility neuroprostheses. Proceedings of the 20th Annual International Conference of the IEEE Engineering in Medicine and Biology Society; 1998 Oct 28-Nov 1; Hong Kong. p. 2605-8. http://dx.doi.org/10.1109/IEMBS.1998.744991

15. Audu ML, Nataraj R, Gartman SJ, Triolo RJ. Posture shifting after spinal cord injury using functional neuromuscular stimulation - a computer simulation study. J Biomech. 2011; 44(9):1639-45. [PMID:21536290] http://dx.doi.org/10.1016/j.jbiomech.2010.12.020

16. Nataraj R, Audu ML, Kirsch RF, Triolo RJ. Trunk acceleration for neuroprosthetic control of standing: A pilot study. J Appl Biomech. 2012;28(1):85-92. [PMID:21975251]

17. Kobetic R, Marsolais EB, Miller PC. Function and strength of electrically stimulated hip flexor muscles in paraplegia. IEEE Trans Rehabil Eng. 1994;2(1):11-17. http://dx.doi.org/10.1109/86.296347

18. Winter DA. Biomechanics and motor control of human movement. 2nd ed. New York (NY): Wiley; 1990. p. 56-57. 
19. Gill PE, Murray W, Saunders MA. User's guide for SNOPT version 6, a Fortran package for large-scale nonlinear programming. La Jolla (CA): Stanford University; 2005 Aug 28. Available from: https://extras.csc.fi/math/comsol/3.4/doc/ optlab/sndoc7.pdf

20. Hawes MR, Sovak D. Quantitative morphology of the human foot in a North American population. Ergonomics. 1994;37(7):1213-26. [PMID:8050406] http://dx.doi.org/10.1080/00140139408964899

21. Erdemir A, McLean S, Herzog W, van den Bogert AJ. Model-based estimation of muscle forces exerted during movements. Clin Biomech (Bristol, Avon). 2007;22(2): 131-54. [PMID:17070969] http://dx.doi.org/10.1016/j.clinbiomech.2006.09.005

22. Tsuji T, Morasso PG, Goto K, Ito K. Human hand impedance characteristics during maintained posture. Biol Cybern. 1995;72(6):475-85. [PMID:7612720] http://dx.doi.org/10.1007/BF00199890

23. Dolan JM, Friedman MB, Nagurka ML. Dynamic and loaded impedance components in the maintenance of human arm posture. IEEE Trans Syst Man Cybern. 1993; 23(3):698-709. http://dx.doi.org/10.1109/21.256543

24. Dyck M, Jazayeri A, Tavakoli M. Is the human operator in a teleoperation system passive? Proceedings of the World Haptics Conference; 2013 Apr 14-17; Daejeon, Korea. p. 683-88.

25. Rancourt D, Hogan N. Dynamics of pushing. J Mot Behav. 2001;33(4):351-62. [PMID:11734410] http://dx.doi.org/10.1080/00222890109601919

26. Nataraj R, Audu ML, Kirsch RF, Triolo RJ. Center of mass acceleration feedback control for standing by functional neuromuscular stimulation: A simulation study. J Rehabil Res Dev. 2012;49(2):279-96. [PMID:22773529] http://dx.doi.org/10.1682/JRRD.2010.12.0235
27. Nataraj R, Audu ML, Kirsch RF, Triolo RJ. Comprehensive joint feedback control for standing by functional neuromuscular stimulation-a simulation study. IEEE Trans Neural Syst Rehabil Eng. 2010;18(6):646-57. [PMID:20923741] http://dx.doi.org/10.1109/TNSRE.2010.2083693

28. Visioli A. Practical PID control. London (England): Springer; 2006.

29. Griffin JD, Kolda TG, Lewis RM. Asynchronous parallel generating set search for linearly-constrained optimization. SIAM J Sci Comput. 2008;30(4):1892-1924. http://dx.doi.org/10.1137/060664161

30. Nataraj R, Audu ML, Triolo RJ. Comparing joint kinematics and center of mass acceleration as feedback for control of standing balance by functional neuromuscular stimulation. J Neuroeng Rehabil. 2012;9;25. [PMID:22559852] http://dx.doi.org/10.1186/1743-0003-9-25

Submitted for publication June 25, 2013. Accepted in revised form November 15, 2013.

This article and any supplementary material should be cited as follows:

Audu ML, Gartman SJ, Nataraj N, Triolo RJ. Posturedependent control of stimulation in standing neuroprosthesis: Simulation feasibility study. J Rehabil Res Dev. 2014;51(3):481-96.

http://dx.doi.org/10.1682/JRRD.2013.06.0150

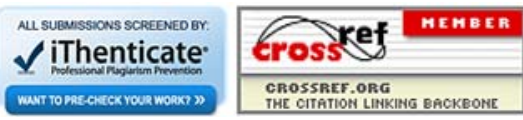

